Kukush, Malenko, Schneeweiss, Shalabh:

Optimality of Quasi-Score in the multivariate mean-variance model with an application to the zero-inflated Poisson model with measurement errors

Sonderforschungsbereich 386, Paper 498 (2006)

Online unter: http://epub.ub.uni-muenchen.de/

Projektpartner
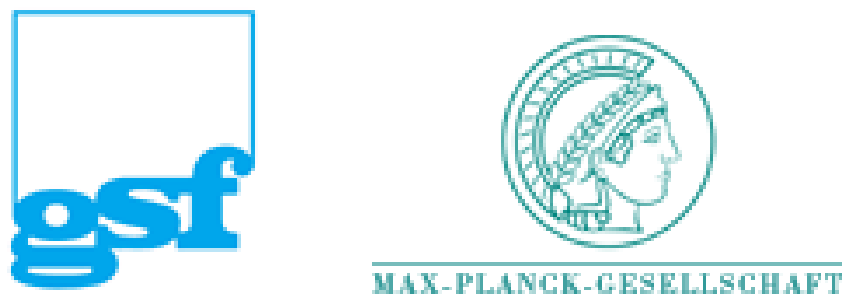


\title{
Optimality of Quasi-Score in the multivariate mean-variance model with an application to the zero-inflated Poisson model with measurement errors
}

\author{
Alexander Kukush ${ }^{1}$, Andrii Malenko ${ }^{2}$, Hans Schneeweiss ${ }^{3}$, and Shalabh ${ }^{4}$ \\ SFB Discussion Paper 498 \\ University of Munich, Munich (Germany)
}

\begin{abstract}
In a multivariate mean-variance model, the class of linear score (LS) estimators based on an unbiased linear estimating function is introduced. A special member of this class is the (extended) quasi-score (QS) estimator. It is "extended" in the sense that it comprises the parameters describing the distribution of the regressor variables. It is shown that QS is (asymptotically) most efficient within the class of LS estimators. An application is the multivariate measurement error model, where the parameters describing the regressor distribution are nuisance parameters. A special case is the zero-inflated Poisson model with measurement errors, which can be treated within this framework.
\end{abstract}

Keywords: Multivariate mean-variance model, measurement errors, zero-inflated Poisson model, nuisance parameters, quasi-score, linear score, corrected score, asymptotic efficiency.

\footnotetext{
${ }^{1}$ Faculty of Mechanics and Mathematics, Kyiv National Taras Shevchenko University, Volodymyrska st. 64, 01033, Kyiv (Ukraine), E-mail: alexander_kukush@univ.kiev.ua

${ }^{2}$ Faculty of Mechanics and Mathematics, Kyiv National Taras Shevchenko University, Volodymyrska st. 64, 01033, Kyiv (Ukraine), E-mail: exipilis@yandex.ru

${ }^{3}$ Department of Statistics, University of Munich, Akademiestrasse 1, 80799 Munich (Germany), E-mail: Hans.Schneeweiss@stat.uni-muenchen.de

${ }^{4}$ (Corresponding author) Department of Mathematics \& Statistics, Indian Institute of Technology, Kanpur - 208016 (India), E-mail: shalab@iitk.ac.in; shalabh1@yahoo.com
} 


\section{Introduction}

We consider a $q$-dimensional random vector $y$ which is related to a $p$-dimensional random vector $x$ via a conditional vector valued mean function $m(x, \theta):=\mathbf{E}(y \mid x)$. The mean function depends on an unknown $d$-dimensional parameter vector $\theta$ to be estimated with the help of an iid sample $\left(x_{i}, y_{i}\right), i=\overline{1, n}$. (All vectors are taken to be column vectors). The mean function is supplemented by a matrix valued conditional variance function $v(x, \theta):=\mathbf{V}(y \mid x)$ depending on the same parameter vector $\theta$ as the mean function. This parameter vector also determines the distribution of the regressor variable $x$, which is supposed to be given by a density function $\rho(x, \theta)$. Such a model may arise in the context of measurement error models.

We can estimate $\theta$ by constructing a quasi-score function. However the usual quasi-score function

$$
\frac{\partial m^{\top}(x, \theta)}{\partial \theta} v(x, \theta)^{-1}\{y-m(x, \theta)\}
$$

cf., e.g., Armstrong (1985), Carroll et al. (2006), Heyde (1997), Wedderburn (1974), is not optimal and sometimes not even feasible. We extend the quasi-score function by adding the term $\partial \log \rho(x, \theta) / \partial \theta$. This extended quasi-score function is again called the quasi-score (QS) function of the model.

We show that the QS estimator of $\theta$ based on this QS function is optimal within the class of so-called linear score (LS) estimators, which are based on linearin- $y$ unbiased estimating (or: score) functions. Optimality is defined in terms of the asymptotic covariance matrices (ACMs) of the QS and LS estimators. We also derive a formula for the rank of the difference of the two ACMs.

This paper is a generalization of some of the results of Kukush et al. (2006) to the case of a multivariate response variable $y$, whereas in the previous paper only the univariate case was considered. The proofs, however, carry over with 
only minor changes and will therefore be omitted.

An application of the multivariate model is the zero-inflated log-linear Poisson measurement error model, which is characterized by the property that the distribution of a count variable $y$ is given by a Poisson law for $y>0$, while the value $y=0$ occurs with a separate probability unrelated to the Poisson distribution, cf., e.g., Cameron and Trivedi (1998), Czado and Min (2006), Lambert(1992). Although this model is univariate it can be studied under the guise of a two-dimensional multivariate model, where the indicator variable for the event $y=0$ serves as the second variable.

In the following, we often suppress the arguments in the various functions. E.g., we write $m$ instead of $m(x, \theta)$. Derivatives with respect to $\theta$ (or other variables) are denoted by a subscript, e.g., $(\log \rho)_{\theta}:=\partial \log \rho(x, \theta) / \partial \theta$, which is a vector of the same dimension as $\theta$. For a vector, like $m$, the derivative $m_{\theta}$ is a matrix (i.e., $\left.m_{\theta}:=\partial m / \partial \theta^{\top}\right)$, and for a matrix, it is a tensor. E.g., if $g$ is a $(d \times q)$ matrix with elements $g_{i j}, i=\overline{1, d}, j=\overline{1, q}$, then $g_{\theta}$ is a tensor with elements $g_{i k}^{j}:=$ $\partial g_{i j} / \partial \theta_{k}, k=\overline{1, d}$, such that $g_{\theta} y$ is a matrix with elements $\left(g_{\theta} y\right)_{i k}=\sum_{j=1}^{d} g_{i k}^{j} y_{j}$, so that $(g y)_{\theta}=g_{\theta} y$.

Section 2 introduces the LS and QS estimators in a general mean-variance model and states the main results on the optimality of QS. Section 3 applies the general theory to the zero-inflated Poison measurement error model. Section 4 has some simulation results, and Section 5 concludes. 


\section{The general mean-variance model}

\subsection{LS and QS estimators of a mean-variance model}

Let $x$ and $y$ be random vectors distributed in $\mathbb{R}^{p}$ and $\mathbb{R}^{q}$, respectively. Conditional mean and conditional variance of $y$ given $x$ are supposed to be known except for an unknown parameter vector $\theta$ with dimension $d$ :

$$
m(x, \theta)=\mathbf{E}(y \mid x) \in \mathbb{R}^{q}, \quad v(x, \theta)=\mathbf{V}(y \mid x) \in \mathbb{R}^{q \times q} .
$$

We assume that $v(x, \theta)$ is a positive definite matrix for all $x$ and $\theta$. Let $x$ have marginal density $\rho(x, \theta)$.

The class $\mathcal{L}$ of all unbiased linear-in- $y$ scores consists of functions

$$
S_{L}(x, y ; \theta)=g(x, \theta) y-h(x, \theta)
$$

where $g$ is a matrix of size $d \times q$ and $h$ is a vector of dimension $d$. Unbiasedness means that, for all $\theta, \mathbf{E} S_{L}(x, y ; \theta)=0$. Note that the expectation of a random function of $\theta$ is always taken under the same value of $\theta$ as the $\theta$ in the argument of the function.

Suppose an iid sample $\left(x_{i}, y_{i}\right), i=\overline{1, n}$, is given. The LS estimator $\hat{\theta}_{L}$ based on $S_{L}$ is given by the solution to the equation

$$
\sum_{i=1}^{n} S_{L}\left(x_{i}, y_{i} ; \hat{\theta}_{L}\right)=0 .
$$

Under regularity conditions, as detailed for a similar model in Kukush and Schneeweiss (2005), see also Schervish (1995), the solution $\hat{\theta}_{L}$ is, with probability tending to 1 , unique for sufficiently large $n$ and $\hat{\theta}_{L}$ is consistent and asymptotically normal with an ACM given by

$$
\Sigma_{L}=\left(\mathbf{E} S_{L \theta}\right)^{-1} \mathbf{E}\left(S_{L} S_{L}^{\top}\right)\left(\mathbf{E} S_{L \theta}\right)^{-\top}
$$


The most important regularity condition is the condition that $\mathbf{E} S_{L \theta}$ should be nonsingular. We call this the identifiability condition.

Quasi Score is a particular element of the class $\mathcal{L}$. It is given by the QS function

$$
S_{Q}(x, y ; \theta)=m_{\theta}^{\top} v^{-1}(y-m)+(\log \rho)_{\theta} .
$$

Under regularity conditions, see Kukush and Schneeweiss (2005), $\hat{\theta}_{Q}$ is consistent and asymptotically normal with the $\operatorname{ACM} \Sigma_{Q}=\left(\mathbf{E} S_{Q} S_{Q}^{\top}\right)^{-1}$. The identifiability condition here boils down to the condition that $\mathbf{E} S_{Q} S_{Q}^{\top}=\mathbf{E} m_{\theta}^{\top} v^{-1} m_{\theta}+$ $\mathbf{E}(\log \rho)_{\theta}(\log \rho)_{\theta}^{\top}$ should be positive definite. This is equivalent to the condition that the system of $(q+1)$-dimensional random vectors

$$
\left\{m_{1 \theta_{k}}, m_{2 \theta_{k}}, \cdots, m_{q \theta_{k}},(\log \rho)_{\theta_{k}}, k=\overline{1, d}\right\} \quad \text { is linearly independent, }
$$

where $m=\left(m_{1}, m_{2}, \cdots, m_{q}\right)^{\top}$.

\subsection{Optimality of QS}

The following identity is useful in proving the optimality of QS within the class $\mathcal{L}:$

$$
\mathbf{E} S_{L \theta}+\mathbf{E} S_{L} S_{Q}^{\top}=0 .
$$

To prove (4), first note that

$$
\begin{aligned}
\mathbf{E} S_{L} S_{Q}^{\top} & =\mathbf{E} g(y-m)(y-m)^{\top} v^{-1} m_{\theta}+\mathbf{E}(g m-h)(\log \rho)_{\theta}^{\top} \\
& =\mathbf{E} g m_{\theta}+\mathbf{E}(g m-h)(\log \rho)_{\theta}^{\top} .
\end{aligned}
$$

In addition, by differentiating $\mathbf{E} S_{L}=\mathbf{E}(g m-h)$, which is identically equal to zero, with respect to $\theta$, we obtain the identity

$$
\mathbf{E}(g m-h)_{\theta}+\mathbf{E}(g m-h)(\log \rho)_{\theta}^{\top}=0 .
$$


Now,

$$
\mathbf{E} S_{L \theta}=\mathbf{E}\left(g_{\theta} m-h_{\theta}\right),
$$

where $g_{\theta}$ is a tensor, see Section 1, and (4) holds as a consequence of (5) and (6).

In a similar way as in Kukush et al. (2006), we can prove the following theorem by applying (4).

Theorem 2.1 In a mean-variance model,

$$
\Sigma_{L} \geq \Sigma_{Q}
$$

in the sense of the Löewner order.

More details are provided by the following theorem.

Theorem 2.2 In a mean-variance model,

$$
\operatorname{rank}\left(\Sigma_{L}-\Sigma_{Q}\right)=\operatorname{rank}\left[\left(\begin{array}{c}
(g v)_{i 1} \\
\vdots \\
(g v)_{i q} \\
(g m-h)_{i}
\end{array}\right),\left(\begin{array}{c}
\left(m_{1}\right)_{\theta_{i}} \\
\vdots \\
\left(m_{q}\right)_{\theta_{i}} \\
(\log \rho)_{\theta_{i}}
\end{array}\right), i=\overline{1, d}\right]-d .
$$

Proof: Kukush et al. (2006), proof of Theorem 4.2, have shown that

$$
\operatorname{rank}\left(\Sigma_{L}-\Sigma_{Q}\right)=\operatorname{rank}\left\{\left(S_{L}\right)_{i}, \quad\left(S_{Q}\right)_{i}, i=\overline{1, d}\right\}-d
$$

The rank on the r.h.s. of (8) can be expressed in terms of the constituents of $S_{L}$ and $S_{Q}$. For this purpose, we evaluate the defect of the system of random variables $\left\{\left(S_{L}\right)_{i},\left(S_{Q}\right)_{i}, i=\overline{1, d}\right\}$, which is the maximum number of linearly independent constant vectors $\left(c_{1}^{\top}, c_{2}^{\top}\right)^{\top}$ which satisfy the equation

$$
c_{1}^{\top} S_{L}+c_{2}^{\top} S_{Q}=0, \text { a.s, }
$$


or, equivalently,

$$
c_{1}^{\top}(g y-h)+c_{2}^{\top}\left[m_{\theta}^{\top} v^{-1}(y-m)+(\log \rho)_{\theta}\right]=0, \text { a.s. }
$$

By similar arguments as in Kukush et al. (2006), using the condition that $v$ is positive definite for all $x$, this equation can be rewritten as a system of two equations, one concerning the terms pertaining to $y$, the other one concerning the remaining terms:

$$
\begin{aligned}
c_{1}^{\top} g v+c_{2}^{\top} m_{\theta}^{\top} & =0, \\
c_{1}^{\top}(g m-h)+c_{2}^{\top}(\log \rho)_{\theta} & =0,
\end{aligned}
$$

a.s. Thus

$$
\operatorname{def}\left\{\left(S_{L}\right)_{i},\left(S_{Q}\right)_{i}, i=\overline{1, d}\right\}=\operatorname{def}\left[\left(\begin{array}{c}
(g v)_{i 1} \\
\vdots \\
(g v)_{i q} \\
(g m-h)_{i}
\end{array}\right),\left(\begin{array}{c}
\left(m_{1}\right)_{\theta_{i}} \\
\vdots \\
\left(m_{q}\right)_{\theta_{i}} \\
(\log \rho)_{\theta_{i}}
\end{array}\right), i=\overline{1, d}\right]
$$

As both systems in (9) have the same number, $2 d$, of random elements (on the lefthand side random variables, on the right-hand side random vectors), the equality of defects implies the equality of ranks. The statement of the theorem now follows from (8) and (9).

\subsection{Marginal Quasi Score}

Starting from a multivariate mean-variance model, we can always consider a subvector of $y$ and set up the corresponding marginal mean-variance model for this subvector. In particular, the subvector may consist of a single component of $y$. We can construct a marginal quasi-score function with this marginal model. As long as the identifiability condition (3) for this marginal QS function is satisfied, we can use it to estimate $\theta$. 
We study the relation between the full and the marginal QS estimator of $\theta$. For simplicity, let $q=2$. We consider the marginal QS estimator which uses only $y_{1}$ and is based on the marginal QS function

$$
S_{Q^{*}}=\frac{m_{1 \theta}\left(y_{1}-m_{1}\right)}{v_{11}}+(\log \rho)_{\theta} .
$$

This estimator is most efficient in the class of estimators based on a linear-in- $y_{1}$ estimating function. Above we considered estimators linear in $\left(y_{1}, y_{2}\right)^{\top}$, and it is obvious from Theorem 2.1 that

$$
\Sigma_{Q^{*}} \geq \Sigma_{Q}
$$

We can compute the rank of $\Sigma_{Q^{*}}-\Sigma_{Q}$. Consider the functions

$$
\begin{aligned}
g^{*} & =\left(\frac{m_{1 \theta}}{v_{11}}, 0\right), \\
h^{*} & =\frac{m_{1 \theta} m_{1}}{v_{11}}-(\log \rho)_{\theta} .
\end{aligned}
$$

Then $S_{Q^{*}}=g^{*} y-h^{*}$. Furthermore,

$$
\begin{aligned}
g^{*} v & =\left(m_{1 \theta}, \frac{v_{12}}{v_{11}} m_{1 \theta}\right), \\
g^{*} m-h^{*} & =(\log \rho)_{\theta} .
\end{aligned}
$$

By $(7), \operatorname{rank}\left(\Sigma_{Q^{*}}-\Sigma_{Q}\right)+d=$

$$
\operatorname{rank}\left[\begin{array}{cc}
m_{1 \theta}^{\top} & m_{1 \theta}^{\top} \\
m_{1 \theta}^{\top} v_{12} v_{11}^{-1} & m_{2 \theta}^{\top} \\
(\log \rho)_{\theta}^{\top} & (\log \rho)_{\theta}^{\top}
\end{array}\right]=\operatorname{rank}\left[\begin{array}{cc}
0 & m_{1 \theta}^{\top} \\
m_{1 \theta}^{\top} v_{12} v_{11}^{-1}-m_{2 \theta}^{\top} & m_{2 \theta}^{\top} \\
0 & (\log \rho)_{\theta}^{\top}
\end{array}\right] .
$$

As by assumption $v(x, \theta)$ is positive definite for all $x$, therefore $v_{11}$ is positive for all $x$ and hence

$$
\operatorname{rank}\left(\Sigma_{Q^{*}}-\Sigma_{Q}\right)=\operatorname{rank}\left[\begin{array}{cc}
0 & m_{1 \theta}^{\top} \\
m_{1 \theta}^{\top} v_{12}-m_{2 \theta}^{\top} v_{11} & m_{2 \theta}^{\top} v_{11} \\
0 & (\log \rho)_{\theta}^{\top}
\end{array}\right]-d
$$

where $\operatorname{rank}[\cdot]$ is the column rank of the system of random variables [.]. 


\section{Zero-Inflated Poisson model}

\subsection{The model and its QS estimator}

Consider a scalar response variable $y_{1}$ and a scalar regressor variable $\xi$ such that the conditional distribution of $y_{1}$ given $\xi$ is a mixture of a Poisson distribution $\operatorname{Po}(\eta)$ with parameter $\eta$ and a one-point distribution $\delta_{0}$ at point zero with mixing parameter $\alpha \in(0,1)$ :

$$
y_{1} \mid \xi \sim \alpha \delta_{0}+(1-\alpha) \operatorname{Po}(\eta) .
$$

Let $\eta=\exp \left(\beta_{0}+\beta_{1} \xi\right)$. In addition to $y_{1}$, we introduce the indicator variable

$$
y_{2}=I\left(y_{1}=0\right)
$$

so that $y=\left(y_{1}, y_{2}\right)^{\top}$ is a bivariate response variable. This zero-inflated log-linear Poisson model is a special case of our general model with $p=1$ and $q=2$. The distribution of $y \mid \xi$ is given by

$$
p(y \mid \xi)=(1-\alpha) \frac{e^{-\eta} \eta^{y_{1}}}{y_{1} !}\left(1+\frac{\alpha}{1-\alpha} e^{\eta}\right)^{y_{2}} .
$$

The variable $\xi$ is not directly observable. Instead we observe

$$
x=\xi+\delta
$$

with a measurement error $\delta \sim N\left(0, \sigma_{\delta}^{2}\right), \sigma_{\delta}^{2}>0$, which is independent of $\xi$ and $y$. The error variance $\sigma_{\delta}^{2}$ is assumed to be known. In addition, we assume $\xi \sim N\left(\mu, \sigma_{\xi}^{2}\right), \sigma_{\xi}^{2}>0$, so that

$$
\log \rho(x, \theta)=-\frac{(x-\mu)^{2}}{2 \sigma^{2}}-\log \sigma+\text { const }, \quad \sigma^{2}=\sigma_{\xi}^{2}+\sigma_{\delta}^{2} .
$$

The unknown parameter vector of this model is $\theta=\left(\alpha, \beta_{0}, \beta_{1}, \mu, \sigma\right)^{\top}$, and thus $d=5$. To derive the mean-variance model for $y \mid x$, we need to compute $\mu_{1}(x):=$ $\mathbf{E}(\xi \mid x)$ and $\tau^{2}:=\mathbf{V}(\xi \mid x)$. We have

$$
\mu_{1}(x)=K x+(1-K) \mu \text { with } K=K(\sigma)=1-\sigma_{\delta}^{2} \sigma^{-2} \text { and } \tau^{2}=\sigma_{\delta}^{2} K,
$$


where $K$ is the reliability ratio, cf. Kukush et al. (2006), Section 6.2. The mean function $m(x, \theta)=\left(m_{1}(x, \theta), m_{2}(x, \theta)\right)^{\top}$ is given by

$$
\begin{aligned}
& m_{1}(x, \theta)=(1-\alpha) \exp \left\{\beta_{0}+\mu_{1}(x) \beta_{1}+\beta_{1}^{2} \tau^{2} / 2\right\} \\
& m_{2}(x, \theta)=\alpha+(1-\alpha) \mathbf{E}(f \mid x)
\end{aligned}
$$

where $f=f(t)=\exp \left\{-e^{t}\right\}$ with $t=\beta_{0}+\beta_{1} \mu_{1}(x)+\beta_{1} \tau \gamma$ and $\gamma \sim N(0,1)$, independent of $x$. The matrix $v=v(x, \theta)$ is expressed in terms of $m_{1}$ and $m_{2}$ as follows:

$$
v=\left(\begin{array}{cc}
m_{1}\left(1-m_{1}\right)+\frac{1}{1-\alpha} e^{\beta_{1}^{2} \tau^{2}} m_{1}^{2} & -m_{1} m_{2} \\
-m_{1} m_{2} & m_{2}\left(1-m_{2}\right)
\end{array}\right)
$$

With these mean and variance functions, we can set up the QS estimator as in Section 2.1.

It can be proved that $v(x, \theta)$ is p.d. for all $x$ and $\theta$, a.s. Indeed, we have

$$
v(x, \theta)=\mathbf{E}(\mathbf{V}(y \mid \xi) \mid x)+\mathbf{V}(\mathbf{E}(y \mid \xi) \mid x) \geq \mathbf{E}(\mathbf{V}(y \mid \xi) \mid x)
$$

and it is enough to show, that $\mathbf{V}(y \mid \xi)$ is p.d. for all $\xi$ and $\theta$ a.s. Let $z$ be an indicator variable independent of $\xi$, with $P(z=0)=\alpha$ and $P(z=1)=1-\alpha$, such that $y_{1} \mid(\xi, z=1) \sim \operatorname{Po}(\eta)$ and $y_{1} \mid(\xi, z=0) \sim \delta_{0}$. Then

$$
\mathbf{V}(y \mid \xi) \geq \mathbf{E} \mathbf{V}(y \mid \xi, z)=(1-\alpha) \mathbf{V}(y \mid \xi, z=1)=(1-\alpha)\left(\begin{array}{cc}
\eta & -\eta e^{-\eta} \\
-\eta e^{-\eta} & e^{-\eta}\left(1-e^{-\eta}\right)
\end{array}\right)
$$

which is positive definite.

We can prove that the QS estimator of $\mu$ is just the empirical mean. Indeed, 
consider $m_{\theta}$ :

$$
\begin{aligned}
m_{1 \theta} & =\left(-\frac{1}{1-\alpha}, 1, \mu_{1}(x)+\tau^{2} \beta_{1},(1-K) \beta_{1}, \beta_{1}(x-\mu) \frac{\partial K}{\partial \sigma}+\frac{1}{2} \beta_{1}^{2} \frac{\partial \tau^{2}}{\partial \sigma}\right)^{\top} m_{1}, \\
m_{2 \alpha} & =1-\mathbf{E}(f \mid x), \\
m_{2 \beta_{0}} & =(1-\alpha) \mathbf{E}\left(f^{\prime} \mid x\right), \\
m_{2 \beta_{1}} & =(1-\alpha)\left(\mu_{1}(x) \mathbf{E}\left(f^{\prime} \mid x\right)+\beta_{1} \tau^{2} \mathbf{E}\left(f^{\prime \prime} \mid x\right)\right) \\
m_{2 \mu} & =(1-\alpha)(1-K) \beta_{1} \mathbf{E}\left(f^{\prime} \mid x\right), \\
m_{2 \sigma} & =(1-\alpha) \beta_{1}\left((x-\mu) \frac{\partial K}{\partial \sigma} \mathbf{E}\left(f^{\prime} \mid x\right)+\frac{1}{2} \beta_{1} \frac{\partial \tau^{2}}{\partial \sigma} \mathbf{E}\left(f^{\prime \prime} \mid x\right)\right) .
\end{aligned}
$$

Here, we used the identity

$$
\mathbf{E}\left[\gamma f^{\prime}(a(x)+c \gamma) \mid x\right]=c \mathbf{E}\left[f^{\prime \prime}(a(x)+c \gamma) \mid x\right]
$$

with $c=\beta_{1} \tau$. We see that

$$
\left(m_{1 \mu}, m_{2 \mu}\right)=(1-K) \beta_{1}\left(m_{1 \beta_{0}}, m_{2 \beta_{0}}\right)
$$

This implies that from the second and fourth equations for $\hat{\theta}_{Q}$, i.e., from

$$
\begin{aligned}
\sum_{i=1}^{n}\left\{m_{1 \beta_{0}}\left(x_{i}, \theta\right), m_{2 \beta_{0}}\left(x_{i}, \theta\right)\right\} v^{-1}\left\{y_{i}-m\left(x_{i}, \theta\right)\right\} & =0, \\
\sum_{i=1}^{n}\left\{m_{1 \mu}\left(x_{i}, \theta\right), m_{2 \mu}\left(x_{i}, \theta\right)\right\} v^{-1}\left\{y_{i}-m\left(x_{i}, \theta\right)\right\}+\sum_{i=1}^{n}\left\{\log \rho\left(x_{i}, \theta\right)\right\}_{\mu} & =0,
\end{aligned}
$$

we obtain $\hat{\mu}_{Q}=\bar{x}$.

On the other hand, the QS estimator of $\sigma^{2}$ is not the empirical variance of $x_{i}, i=\overline{1, n}$. We will give an indirect proof of this fact in the next section.

\section{Note}

It is interesting to note that a marginal QS method, which uses only the conditional mean and variance of $y_{1}$ does not work. Indeed, such a method would be 
based on the quasi score function

$$
S_{Q^{*}}=m_{1 \theta} v_{11}^{-1}\left(y_{1}-m_{1}\right)+(\log \rho)_{\theta},
$$

alone, see (10). But since the first two components of $m_{1 \theta}$ are linearly dependent, see (14), the estimating equations based on $S_{Q^{*}}$ are not sufficient to produce a unique solution $\hat{\theta}_{Q^{*}}$. Looked at it from another angle, it is seen that the identifiability condition (3) is violated.

\subsection{Modified Corrected Score}

In this section we construct a score function to estimate $\theta$, which does not use any information about the distribution of $x$.

Consider the ML score for $(\alpha, \beta)$ in the error-free model:

$$
S_{M L}=\frac{y_{1}}{\eta}\left(\begin{array}{c}
0 \\
\eta_{\beta}
\end{array}\right)+\frac{y_{2}}{\alpha+(1-\alpha) e^{-\eta}}\left(\begin{array}{c}
\frac{1}{1-\alpha} \\
\alpha \eta_{\beta}
\end{array}\right)-\left(\begin{array}{c}
\frac{1}{1-\alpha} \\
\eta_{\beta}
\end{array}\right), \quad \eta_{\beta}=\eta\left(\begin{array}{c}
1 \\
\xi
\end{array}\right) .
$$

It is not possible to construct the so-called corrected score function $S_{C}^{(\alpha, \beta)}$ as the solution to the deconvolution problem

$$
\mathbf{E}\left(S_{C}^{(\alpha, \beta)}(x, y ; \theta) \mid \xi, y\right)=S_{M L}(\xi, y ; \theta)
$$

cf. Nakamura (1990), because there are complex zeros in the common denominator of $S_{M L}$, cf. Stefanski (1989). Therefore we modify $S_{M L}$ by multiplying the first component of $S_{M L}$ by $(1-\alpha)\left(\alpha+(1-\alpha) e^{-\eta}\right)$ and the other two components by $\alpha+(1-\alpha) e^{-\eta}$. It should be noted that this modified $S_{M L}$ is no more optimal in the context of the error free model. Nevertheless, we use it to construct a modified score $S_{C}^{(\alpha, \beta)}$, which is the solution to the modified deconvolution problem

$$
\mathbf{E}\left(S_{C}^{(\alpha, \beta)}(x, y ; \theta) \mid \xi, y\right)=\left(\alpha+(1-\alpha) e^{-\eta}\right) \operatorname{diag}\{1-\alpha, 1,1\} S_{M L}(\xi, y ; \theta) .
$$


The parameters $\mu$ and $\sigma^{2}$ are estimated as empirical mean and variance, respectively.

The modified corrected score $S_{C}$ is a linear unbiased score function, $S_{C}=$ $g y-h$, where

$$
\mathbf{E}(g \mid \xi)=\left(\begin{array}{cc}
0 & 1 \\
\alpha+(1-\alpha) e^{-\eta} & \alpha \eta \\
\left(\alpha+(1-\alpha) e^{-\eta}\right) \xi & \alpha \eta \xi \\
0 & 0 \\
0 & 0
\end{array}\right), \quad \mathbf{E}(h \mid \xi)=\left(\begin{array}{c}
\alpha+(1-\alpha) e^{-\eta} \\
\left(\alpha+(1-\alpha) e^{-\eta}\right) \eta \\
\left(\alpha+(1-\alpha) e^{-\eta}\right) \eta \xi \\
\xi-\mu \\
(\xi-\mu)^{2}-\sigma_{\xi}^{2}
\end{array}\right)
$$

The last two components of $h$ are

$$
\begin{aligned}
& h_{4}=x-\mu, \\
& h_{5}=(x-\mu)^{2}-\sigma^{2} .
\end{aligned}
$$

The other components of $h$ and the elements of $g$ are given below, see Section 4 .

The estimator based on this score function is the modified corrected score (MCS) estimator.

The following theorem states the efficiency of the QS estimator vis-à-vis the MCS estimator as measured by the difference of the ACMs.

Theorem 3.1 Under $\alpha \in(0,1), \beta_{1} \neq 0$,

$$
\Sigma_{Q}^{(\alpha, \beta, \sigma)}<\Sigma_{C}^{(\alpha, \beta, \sigma)}
$$

These matrices are the ACMs of the $Q S$ and MCS estimators of $\left(\alpha, \beta_{0}, \beta_{1}, \sigma\right)^{\top}$, respectively. Under $\beta_{1}=0$ and $\alpha \in(0,1)$ we have $\operatorname{rank}\left(\Sigma_{C}^{(\alpha, \beta, \sigma)}-\Sigma_{Q}^{(\alpha, \beta, \sigma)}\right)=1$.

It follows that, under $\beta_{1} \neq 0$, we have $\Sigma_{Q}^{(\sigma)}<\Sigma_{C}^{(\sigma)}$, therefore $\hat{\sigma}_{Q}^{2}$ is not the empirical variance. 
If $\alpha$ is known, then, under $\beta_{1} \neq 0$, the QS estimator of $\left(\beta_{0}, \beta_{1}, \sigma\right)$ is strictly more efficient than the MCS estimator.

\subsection{Proof of Theorem 3.1}

To prove the statement of the Theorem we compute the rank of the system in (7). First note that $\hat{\mu}_{C}=\hat{\mu}_{Q}=\bar{x}$. Therefore from the inequality $\Sigma_{C} \geq \Sigma_{Q}$ we have

$$
\Sigma_{C}-\Sigma_{Q}=\left(\begin{array}{cc}
\Sigma_{C}^{(\alpha, \beta, \sigma)}-\Sigma_{Q}^{(\alpha, \beta, \sigma)} & 0 \\
0 & 0
\end{array}\right) .
$$

The right-hand side of (7) can be written as the rank of the following system of three-dimensional random vectors minus 5:

$$
\left[\begin{array}{ccccccccc}
(g v)_{11} & (g v)_{21} & (g v)_{31} & m_{1 \alpha} & m_{1 \beta_{0}} & m_{1 \beta_{1}} & m_{1 \sigma} & 0 & 0 \\
(g v)_{12} & (g v)_{22} & (g v)_{32} & m_{2 \alpha} & m_{2 \beta_{0}} & m_{2 \beta_{1}} & m_{2 \sigma} & 0 & 0 \\
(g m-h)_{1} & (g m-h)_{2} & (g m-h)_{3} & 0 & 0 & 0 & 0 & x-\mu & (x-\mu)^{2}-\sigma^{2}
\end{array}\right],
$$

where the column $\left(m_{1 \mu}, m_{2 \mu}, 0\right)^{\top}$ was dropped because of $(15)$.

We divide the proof into two parts. First we show that

$$
\left\{(g m-h)_{1},(g m-h)_{2},(g m-h)_{3}, x-\mu,(x-\mu)^{2}-\sigma^{2}\right\}
$$

are linearly independent functions of $x$. Then we show that the functions of $x$

$$
\left\{m_{2 \alpha}, m_{2 \beta_{0}}, m_{2 \beta_{1}}, m_{2 \sigma}\right\}
$$

are linearly independent.

With these two sets of linearly independent functions we immediately obtain that the column rank of the system in $(18)$ is 9 , therefore the rank of $\Sigma_{C}^{(\alpha, \beta, \sigma)}-$ $\Sigma_{Q}^{(\alpha, \beta, \sigma)}$ is 4 , and this matrix is positive definite. 


\subsubsection{Part 1 of the proof}

We want to show that the functions (19) are linearly independent under $\beta_{1} \neq 0$. We consider only the case $\beta_{1}>0$. The case $\beta_{1}<0$ can be treated similarly. We divide the proof into three steps:

1. We prove that $(g m-h)_{i} \rightarrow 0$ as $x \rightarrow-\infty, i=1,2,3$, while $x$ and $x^{2}$ converge to infinity. This means that we can exclude $(x-\mu)$ and $(x-\mu)^{2}-\sigma^{2}$ and consider only the linear independence of $(g m-h)_{i}$.

2. We show that $(g m-h)_{3} \sim x e^{\beta_{1} K x}$, while $(g m-h)_{1,2} \sim e^{\beta_{1} K x}$ as $x \rightarrow-\infty$. This allows us to consider only $(g m-h)_{1}$ and $(g m-h)_{2}$.

3. We show that any linear combination of $(g m-h)_{1}$ and $(g m-h)_{2}$ can be split into two parts with different order of convergence to zero. This will yield linear independence of $(g m-h)_{1}$ and $(g m-h)_{2}$.

Taking all three arguments together, we obtain the linear independence of the total system (19).

However, before we start with these steps, we need to introduce some preliminary considerations. We define functions $u_{i}(x), i=\overline{1,4}$, which are the solutions to the following deconvolution problems:

$$
\mathbf{E}\left(u_{1} \mid \xi\right)=e^{-\eta}, \quad \mathbf{E}\left(u_{2} \mid \xi\right)=e^{-\eta} \xi, \quad \mathbf{E}\left(u_{3} \mid \xi\right)=e^{-\eta} \eta, \quad \mathbf{E}\left(u_{4} \mid \xi\right)=e^{-\eta} \eta \xi
$$


The explicit forms of $u_{k}(x)$ are:

$$
\begin{aligned}
u_{1}(x)= & \sum_{k=0}^{\infty} \frac{(-1)^{k}}{k !} \exp \left\{k \beta_{0}+k \beta_{1} x-k^{2} \beta_{1}^{2} \sigma_{\delta}^{2} / 2\right\} \\
u_{2}(x)= & \sum_{k=0}^{\infty} \frac{(-1)^{k}}{k !}\left(x-k \beta_{1} \sigma_{\delta}^{2}\right) \exp \left\{k \beta_{0}+k \beta_{1} x-k^{2} \beta_{1}^{2} \sigma_{\delta}^{2} / 2\right\} \\
u_{3}(x)= & \sum_{k=0}^{\infty} \frac{(-1)^{k}}{k !} \exp \left\{(k+1) \beta_{0}+(k+1) \beta_{1} x-(k+1)^{2} \beta_{1}^{2} \sigma_{\delta}^{2} / 2\right\} \\
u_{4}(x)= & \sum_{k=0}^{\infty} \frac{(-1)^{k}}{k !}\left\{x-(k+1) \beta_{1} \sigma_{\delta}^{2}\right\} \\
\quad & \quad \times \exp \left\{(k+1) \beta_{0}+(k+1) \beta_{1} x-(k+1)^{2} \beta_{1}^{2} \sigma_{\delta}^{2} / 2\right\} .
\end{aligned}
$$

Due to Fubinis theorem, we can exchange the order of summation and of computing $\mathbf{E}\left(u_{k} \mid \xi\right)$ and can thus check that the functions $u_{k}(x)$ given in (22)-(25) are indeed the solutions to (21). The series in (22)-(25) converge uniformly on $\left(-\infty, x_{0}\right)$ for arbitrary $x_{0} \in \mathbb{R}$. This yields the following asymptotic expansions for $u_{k}$ as $x \rightarrow-\infty$ :

$$
\begin{aligned}
& u_{1}(x)=1-\exp \left\{\beta_{0}+\beta_{1} x-\beta_{1}^{2} \sigma_{\delta}^{2} / 2\right\}+\frac{1}{2} \exp \left\{2 \beta_{0}+2 \beta_{1} x-2 \beta_{1}^{2} \sigma_{\delta}^{2}\right\}+o\left(e^{2 \beta_{1} x}\right), \\
& u_{2}(x)=x+o(x) \\
& u_{3}(x)=\exp \left\{\beta_{0}+\beta_{1} x-\beta_{1}^{2} \sigma_{\delta}^{2} / 2\right\}-\exp \left\{2 \beta_{0}+2 \beta_{1} x-2 \beta_{1}^{2} \sigma_{\delta}^{2}\right\}+o\left(e^{2 \beta_{1} x}\right), \\
& u_{4}(x)=x \exp \left\{\beta_{0}+\beta_{1} x-\beta_{1}^{2} \sigma_{\delta}^{2} / 2\right\}+o\left(x e^{\beta_{1} x}\right) .
\end{aligned}
$$

With the help of the functions $u_{k}(x), k=\overline{1,4}$, we can write expressions for the first three rows of the matrix $g$ and the vector $h$ :

$$
\begin{aligned}
& \left(\begin{array}{l}
g_{1} \\
g_{2} \\
g_{3}
\end{array}\right)=\left(\begin{array}{cc}
0 & 1 \\
\alpha+(1-\alpha) u_{1} & \alpha \exp \left\{\beta_{0}+\beta_{1} x-\beta_{1}^{2} \sigma_{\delta}^{2} / 2\right\} \\
\alpha x+(1-\alpha) u_{2} & \alpha\left(x-\beta_{1} \sigma_{\delta}^{2}\right) \exp \left\{\beta_{0}+\beta_{1} x-\beta_{1}^{2} \sigma_{\delta}^{2} / 2\right\}
\end{array}\right) \\
& \left(\begin{array}{c}
h_{1} \\
h_{2} \\
h_{3}
\end{array}\right)=\left(\begin{array}{c}
\alpha+(1-\alpha) u_{1} \\
\alpha \exp \left\{\beta_{0}+\beta_{1} x-\beta_{1}^{2} \sigma_{\delta}^{2} / 2\right\}+(1-\alpha) u_{3} \\
\alpha\left(x-\beta_{1} \sigma_{\delta}^{2}\right) \exp \left\{\beta_{0}+\beta_{1} x-\beta_{1}^{2} \sigma_{\delta}^{2} / 2\right\}+(1-\alpha) u_{4}
\end{array}\right)
\end{aligned}
$$


Therefore the first three components of the vector $(g m-h)$ are:

$$
\left(\begin{array}{c}
m_{2}-\alpha-(1-\alpha) u_{1} \\
\alpha\left(m_{1}-\left(1-m_{2}\right) \exp \left\{\beta_{0}+\beta_{1} x-\beta_{1}^{2} \sigma_{\delta}^{2} / 2\right\}\right)+(1-\alpha)\left(u_{1} m_{1}-u_{3}\right) \\
\alpha\left(x m_{1}-\left(1-m_{2}\right)\left(x-\beta_{1} \sigma_{\delta}^{2}\right) \exp \left\{\beta_{0}+\beta_{1} x-\beta_{1}^{2} \sigma_{\delta}^{2} / 2\right\}\right)+(1-\alpha)\left(u_{2} m_{1}-u_{4}\right)
\end{array}\right) .
$$

Remember that $m_{1}=$ const $\cdot e^{\beta_{1} K x}$.

We establish the asymptotics of $m_{2}$ as $x \rightarrow-\infty$. Denote $f_{0}=\mathbf{E}(f \mid x)$, where

$$
f=f(t)=\exp \left\{-e^{t}\right\}, \quad t=\beta_{0}+\beta_{1} \mu_{1}(x)+\beta_{1} \tau \gamma, \quad \gamma \sim N(0,1) .
$$

Then $m_{2}=\alpha+(1-\alpha) f_{0}$. Obviously, $t \sim \beta_{1} K x$ and $f(t)=1-e^{t}+\frac{1}{2} e^{2 t}+o\left(e^{2 t}\right)$ as $x \rightarrow-\infty$. By the dominated convergence theorem,

$f_{0}=1-\exp \left\{\beta_{0}+\beta_{1} \mu_{1}(x)+\beta_{1}^{2} \tau^{2} / 2\right\}+\frac{1}{2} \exp \left\{2 \beta_{0}+2 \beta_{1} \mu_{1}(x)+2 \beta_{1}^{2} \tau^{2}\right\}+o\left(e^{2 \beta_{1} K x}\right)$.

It is now easy to see that $m_{2} \rightarrow 1$ as $x \rightarrow-\infty$.

1. Now we are ready for the first step. Consider a linear combination of the functions (19), which is zero for all $x$ :

$$
c_{1}(g m-h)_{1}+c_{2}(g m-h)_{2}+c_{3}(g m-h)_{3}+c_{4}(x-\mu)+c_{5}\left((x-\mu)^{2}-\sigma^{2}\right) \equiv 0 .
$$

From the asymptotic expressions for the functions $m_{1}, m_{2}, u_{1}, \ldots, u_{4}$ it is easily seen that the functions $(g m-h)_{i}$ vanish as $x \rightarrow-\infty, i=\overline{1,3}$. Therefore the coefficients $c_{4}$ and $c_{5}$ in (28) must be equal to zero.

2. Now we establish the asymptotic behavior of $(g m-h)_{i}$. Consider $(g m-h)_{1}$ :

$$
(g m-h)_{1}=(1-\alpha)\left(f_{0}-1\right)+(1-\alpha)\left(1-u_{1}\right) .
$$

As $f_{0}-1 \sim$ const $\cdot e^{\beta_{1} K x}, 1-u_{1} \sim$ const $\cdot e^{\beta_{1} x}$, and $e^{\beta_{1} x}=o\left(e^{\beta_{1} K x}\right)$ we have

$$
(g m-h)_{1} \sim \text { const } \cdot e^{\beta_{1} K x}, \quad x \rightarrow-\infty .
$$


Consider $(g m-h)_{2}$. We have $\left(1-m_{2}\right) e^{\beta_{1} x}=o\left(e^{\beta_{1} K x}\right), u_{3}=o\left(e^{\beta_{1} K x}\right)$, and $u_{1} m_{1} \sim m_{1}$. Therefore

$$
(\mathrm{gm}-h)_{2} \sim m_{1} \sim \mathrm{const} \cdot e^{\beta_{1} K x}, \quad x \rightarrow-\infty .
$$

Consider $(g m-h)_{3}$. We have $\left(1-m_{2}\right)\left(x-\beta_{1} \sigma_{\delta}^{2}\right) e^{\beta_{1} x}=o\left(x e^{\beta_{1} K x}\right), u_{4}=$ $o\left(x e^{\beta_{1} K x}\right)$, and $u_{2} m_{1} \sim x m_{1}$. Therefore

$$
(g m-h)_{3} \sim x m_{1} \sim \text { const } \cdot x e^{\beta_{1} K x}, \quad x \rightarrow-\infty .
$$

We see that $(g m-h)_{1}=o\left((g m-h)_{3}\right)$, and $(g m-h)_{2}=o\left((g m-h)_{3}\right)$ as $x \rightarrow-\infty$. Therefore the coefficient $c_{3}$ in (28) must be equal to zero.

3. Now we can rewrite equation (28) in the equivalent form:

$c_{1}(1-\alpha)\left(f_{0}-u_{1}\right)+c_{2} \alpha\left(m_{1}-(1-\alpha)\left(1-f_{0}\right) \exp \left\{\beta_{0}+\beta_{1} x-\beta_{1}^{2} \sigma_{\delta}^{2} / 2\right\}\right)+c_{2}(1-\alpha)\left(u_{1} m_{1}-u_{3}\right) \equiv 0$.

We rewrite it once again:

$$
\begin{aligned}
& c_{1}(1-\alpha)\left(f_{0}-1\right)+c_{2} m_{1}\left(\alpha+(1-\alpha) u_{1}\right) \equiv \\
& c_{1}(1-\alpha)\left(u_{1}-1\right)+c_{2} \alpha(1-\alpha)\left(1-f_{0}\right) \exp \left\{\beta_{0}+\beta_{1} x-\beta_{1}^{2} \sigma_{\delta}^{2} / 2\right\}+c_{2}(1-\alpha) u_{3} .
\end{aligned}
$$

The left-hand side of (29) is approximated by

$$
\begin{gathered}
a_{1}(1-\alpha)\left(c_{2}-c_{1}\right) e^{\beta_{1} K x}+a_{2}(1-\alpha) c_{1} e^{2 \beta_{1} K x}-a_{3}(1-\alpha)^{2} c_{2} e^{\beta_{1}(K+1) x}+o\left(e^{2 \beta_{1} K x}\right)= \\
a_{1}(1-\alpha)\left(c_{2}-c_{1}\right) e^{\beta_{1} K x}+a_{2}(1-\alpha) c_{1} e^{2 \beta_{1} K x}+o\left(e^{2 \beta_{1} K x}\right), \quad x \rightarrow-\infty,
\end{gathered}
$$

where $a_{i}$ are positive constants. The right-hand side of (29) is approximated by

$$
a_{4}(1-\alpha)\left(c_{2}-c_{1}\right) e^{\beta_{1} x}+\alpha(1-\alpha) c_{2} a_{5} e^{\beta_{1}(K+1) x}+o\left(e^{\beta_{1}(K+1) x}\right), \quad x \rightarrow-\infty,
$$

where $a_{i}$ are also positive constants. We see that (29) is possible only if $c_{1}=c_{2}=$ 0 .

We proved that all the coefficients in (28) are zero, therefore the functions (19) are linearly independent. 


\subsubsection{Part 2 of the proof}

We want to prove that the functions (20) are linearly independent. Due to the expressions for $m_{2 \theta}$ in Section 3.1, we have to prove the linear independence of the functions

$$
\left\{1-f_{0}, f_{1}, \mu_{1}(x) f_{1}+\beta_{1} \tau^{2} f_{2}, \beta_{1}(x-\mu) \frac{\partial K}{\partial \sigma} f_{1}+\frac{1}{2} \beta_{1}^{2} \frac{\partial \tau^{2}}{\partial \sigma} f_{2}\right\}
$$

where we denoted $f_{i}:=\mathbf{E}\left(f^{(i)} \mid x\right)$. This can be transformed into the equivalent set

$$
\left\{1-f_{0}, f_{1}, K x f_{1}+\beta_{1} \tau^{2} f_{2}, \beta_{1} \frac{\partial K}{\partial \sigma} x f_{1}+\frac{1}{2} \beta_{1}^{2} \frac{\partial \tau^{2}}{\partial \sigma} f_{2}\right\}
$$

The last two functions are a linear transformation of the functions $x f_{1}$ and $f_{2}$ with transformation matrix

$$
T=\left(\begin{array}{cc}
K & \beta_{1} \tau^{2} \\
\beta_{1} \frac{\partial K}{\partial \sigma} & \frac{1}{2} \beta_{1}^{2} \frac{\partial \tau^{2}}{\partial \sigma}
\end{array}\right) .
$$

We have

$$
\operatorname{det} T=-\beta_{1}^{2} K \frac{\sigma_{\delta}^{4}}{\sigma^{3}}
$$

which is not zero under $\beta_{1} \neq 0$. Therefore, to prove linear independence of the functions (20), we have to prove linear independence of the functions $\{1-$ $\left.f_{0}, f_{1}, f_{2}, x f_{1}\right\}$.

Consider a linear combination of these functions, which is zero:

$$
c_{0}\left(1-f_{0}\right)+c_{1} f_{1}+c_{2} f_{2}+c_{3} x f_{1} \equiv 0
$$

We establish the asymptotic behavior of the functions in (30) as $x \rightarrow-\infty$. We use the dominated convergence theorem. We have $1-f \sim e^{t}, t \rightarrow-\infty$, and thus for $\gamma \sim N(0,1)$ :

$$
1-f_{0} \sim \mathbf{E}\left(\exp \left\{\beta_{0}+\beta_{1} \mu_{1}(x)+\beta_{1} \tau \gamma\right\} \mid x\right)=\text { const } \cdot e^{\beta_{1} K x}, \quad x \rightarrow-\infty
$$


Consider $f_{1}$. We have $f^{\prime}=-\exp \left\{-e^{t}\right\} e^{t}$ and $f^{\prime} \sim-e^{t}, t \rightarrow-\infty$. Therefore

$$
f_{1} \sim \text { const } \cdot e^{\beta_{1} K x}, \quad x \rightarrow-\infty
$$

Consider $f_{2}$. We have $f^{\prime \prime}=\exp \left\{-e^{t}\right\} e^{2 t}-\exp \left\{-e^{t}\right\} e^{t}$ and $f^{\prime \prime} \sim-e^{t}, t \rightarrow-\infty$. Therefore

$$
f_{2} \sim \text { const } \cdot e^{\beta_{1} K x}, \quad x \rightarrow-\infty
$$

If we divide (30) by $x f_{1}$ and take the limit as $x \rightarrow-\infty$, we see that $c_{3}=0$.

Consider the asymptotics of the functions $f_{i}, i=0,1,2$, as $x \rightarrow+\infty$. We have for arbitrary $a \in \mathbb{R}$ that

$$
\exp \left\{-e^{t}\right\} e^{a t} \rightarrow 0, \quad t \rightarrow \infty
$$

Therefore

$$
f \rightarrow 0, \quad f^{\prime} \rightarrow 0, \quad f^{\prime \prime} \rightarrow 0, \quad t \rightarrow+\infty
$$

and by the dominated convergence theorem

$$
f_{i} \rightarrow 0, \quad i=0,1,2, \quad x \rightarrow+\infty
$$

Thus in equation (30) we have $c_{0}=0$. Now (30) can be rewritten as

$$
\left(c_{1}-c_{2}\right) \mathbf{E}\left(\exp \left\{-e^{t}\right\} e^{t} \mid x\right) \equiv c_{2} \mathbf{E}\left(\exp \left\{-e^{t}\right\} e^{2 t} \mid x\right) .
$$

We see that the asymptotics of the left-hand side and the right-hand side are different as $x \rightarrow-\infty$ because

$$
\exp \left\{-e^{t}\right\} e^{2 t}=o\left(\exp \left\{-e^{t}\right\} e^{t}\right), \quad t \rightarrow-\infty
$$

Therefore $c_{1}=c_{2}=0$, and the functions in (30) are linearly independent. 


\section{Simulations}

A simulation study with the zero-inflated Poisson model of Section 3 was conducted with a threefold objective: first to show that the estimation methods QS and MCS work, at least for large samples, second to corroborate the asymptotic results of the preceding theory, and third to study the behavior of the methods for small samples. A sample size of $n=100$ was taken to be a small sample, while $n=1000$ stood for a large sample. The following parameter values were fixed: $\mu_{\xi}=0.5, \sigma_{\xi}^{2}=0.1, \sigma_{\delta}^{2}=0.1, \alpha=0.6, \beta_{0}=0, \beta_{1}=0.5$. As a variant, $\sigma_{\delta}^{2}=0.05$ was also tried. We simulated $R=1000$ samples $\left(x_{i}, y_{i}\right), i=1, \ldots, n$, of size $n$ and computed the QS and MCS estimates for each sample. Bias and variance were then estimated from the 1000 replications. In addition to QS and MCS, we also computed a naive estimator (NA), which estimates the parameters by ML without taking the measurement errors into account, and, as a benchmark, the ML estimator from the error-free data $\left(\xi_{i}, y_{i}\right), i=1, \ldots, n$, see (16).

For all estimation methods, $\mu$ is estimated by $\hat{\mu}=\bar{x}$. For MCS, $\sigma^{2}$ is estimated by $s_{x}^{2}$, the empirical variance of the sample $x_{i}, i=1, \ldots, n$, while for QS, $\sigma^{2}$ has to be estimated jointly with the other parameters.

For QS, the multivariate conditional mean-variance model given $x$ is set up with (12) as the bivariate mean function and (13) as the covariance matrix. The parameter $\mu$ is replaced with its estimate $\bar{x}$. Deviating from the definition in Section 3, we here denote the main parameter vector $\left(\alpha, \beta_{0}, \beta_{1}\right)^{\top}$ by $\theta$, while $\sigma$ is treated separately.

The QS, estimators of $\theta$ and $\sigma$ are found by applying the method of iteratively reweighted least squares: Let $\theta_{k}$ and $\sigma_{k}$ be the estimated values of $\theta$ and $\sigma$ after the $k$-th iteration. For each sample point $\left(x_{i}, y_{1 i}\right), i=1, \ldots, n$, the vectors and matrices $m\left(x_{i}, \theta_{k}, \sigma_{k}\right), v\left(x_{i}, \theta_{k}, \sigma_{k}\right), M\left(x_{i}, \theta_{k}, \sigma_{k}\right):=\frac{\partial}{\partial \theta^{\top}} m\left(x_{i}, \theta_{k}, \sigma_{k}\right)$ and the 
vector $y_{i}:=\left(y_{1 i}, y_{2 i}\right)$ are computed. The bivariate linear regression

$$
y_{i}-m\left(x_{i}, \theta_{k}, \sigma_{k}\right)=M\left(x_{i}, \theta_{k}, \sigma_{k}\right) d_{k}+u_{i} \quad, i=1, \ldots, n,
$$

with $\operatorname{var}\left(u_{i}\right)=v\left(x_{i}, \theta_{k}, \sigma_{k}\right)$ is set up and is solved for $d_{k}$ by weighted least squares. The value of $\theta$ in the next iteration is then given by $\theta_{k+1}=\theta_{k}+d_{k}$. The value of $\sigma$ in step $k+1$ is found by solving the last equation of the of the system (2), i.e.,

$$
\sum_{i=1}^{n}\left\{m_{1 \sigma}\left(x_{i}\right), m_{2 \sigma}\left(x_{i}\right)\right\} v^{-1}\left(x_{i}\right)\left\{y_{i}-m\left(x_{i}\right)\right\}+\sum_{i=1}^{n}\left\{\log \rho\left(x_{i}\right)\right\}_{\sigma}=0
$$

and is given by

$$
\sigma_{k+1}^{2}=s_{x}^{2}+\frac{1}{n} \sum_{i=1}^{n} w\left(x_{i}, \theta_{k}, \sigma_{k}\right)^{\top} v^{-1}\left(x_{i}, \theta_{k}, \sigma_{k}\right)\left\{y_{i}-m\left(x_{i}, \theta_{k}, \sigma_{k}\right)\right\},
$$

where

$w(x, \theta, \sigma)^{\top}=2 \sigma_{\delta}^{2} \beta_{1}\left(x-\bar{x}+\frac{1}{2} \beta_{1} \sigma_{\delta}^{2},(\alpha-1)\left\{(x-\bar{x}) E\left[b e^{-b} \mid x\right]+\frac{\sigma_{\delta}^{2}}{2 \tau} E\left[b e^{-b} \gamma \mid x\right]\right\}\right)$.

The elements of the matrix $\mathrm{M}$ are given in (14). For the sake of convenience, we repeat the expressions for $m_{2 \theta}$ but in a somewhat different form. Let $b=b(\gamma)=$ $\exp \left(\beta_{0}+\beta_{1} \mu_{1}(x)+\beta_{1} \tau \gamma\right)$, then

$$
\begin{aligned}
m_{2 \alpha} & =1-E\left[e^{-b} \mid x\right] \\
m_{2 \beta_{0}} & =(\alpha-1) E\left[b e^{-b} \mid x\right] \\
m_{2 \beta_{1}} & =(\alpha-1)\left\{\mu_{1}(x) E\left[b e^{-b} \mid x\right]+\tau E\left[b e^{-b} \gamma \mid x\right] .\right.
\end{aligned}
$$

In addition

$$
m_{2 \sigma}=2(\alpha-1) \beta_{1} \frac{\sigma_{\delta}^{2}}{\sigma^{3}}\left\{(x-\bar{x}) E\left[b e^{-b} \mid x\right]+\frac{\sigma_{\delta}^{2}}{2 \tau} E\left[b e^{-b} \gamma \mid x\right]\right\} .
$$

The last two formulae differ from the corresponding formulae in (14) in that the partial integration has not been carried out. Note that $K_{\sigma}=2 \sigma_{\delta}^{2} / \sigma^{3}$ and $\tau_{\sigma}=K_{\sigma} \sigma_{\delta}^{2} /(2 \tau)$. 
For MCS, we have to compute the elements of the first three rows of $g$ and $h$, see (17). These are given by (26) and (27). The infinite series (22)-(25) needed to compute (26) and (27) have been truncated at the order of $k=20$. With $g$ and $h$ so constructed, we can set up the MCS estimating equations:

$$
\sum g\left(x_{i}, \theta\right) y_{i}-\sum h\left(x_{i}, \theta\right)=0
$$

and solve them for $\theta=\left(\alpha, \beta_{0}, \beta_{1}\right)^{\top}$.

The results for $\sigma_{\delta}^{2}=0.1$ are presented in Table 1 . They show that the asymptotic theory is fully corroborated in samples of size $n=1000$. There is only a negligible bias in the three parameter estimates, except, of course, for the naive estimator. The variance of the QS estimates are all smaller than the corresponding ones of the MCS estimates. The variance of the naive estimates are still smaller and even smaller than those of ML, but then these estimates are inconsistent anyway. As compared to the other two parameters of the model, $\alpha$ is estimated very precisely by all estimation methods.

For small samples $(n=100)$, we have similar results, although they are not so clear. Some of the estimates have a small, but noticeable, bias ( e.g., $\hat{\beta}_{0 M C S}$ ), and $\hat{\alpha}_{M C S}$ has a slightly smaller variance than $\hat{\alpha}_{Q S}$. The variances for $n=100$ are a bit more than ten fold the variance for $n=1000$. In $1 \%$ of the runs, the QS estimate could not be computed because of the occurrence of a nearly singular covariance matrix $v$.

When $\sigma_{\delta}^{2}=0.05$, we have similar results both for $n=100$ and $n=1000$, see Table 2. The variances are somewhat smaller than the corresponding ones for $\sigma_{\delta}^{2}=0.01$. For $n=1000$, the difference in the variances of QS and MCS estimates is very small.

As noted above, the variance of $x$ is estimated differently depending on whether QS or MCS is the estimation method. For QS, $\sigma_{x}^{2}$ is estimated along 
Table 1: The bias and variance of $\alpha, \beta_{0}$ and $\beta_{1}$ when $\sigma_{\delta}^{2}=0.1$

\begin{tabular}{|c|c|c|c|c|c|c|}
\hline & \multicolumn{2}{|c|}{$\alpha$} & \multicolumn{2}{c|}{$\beta_{0}$} & \multicolumn{2}{c|}{$\beta_{1}$} \\
\hline Method & Bias & Var & Bias & Var & Bias & Var \\
\hline \multicolumn{7}{|c|}{$n=100$} \\
QS & -0.0258649 & 0.0074825 & -0.0924049 & 0.3186765 & 0.0077364 & 0.7629999 \\
MCS & -0.0247392 & 0.0071527 & -0.1171952 & 0.3455757 & 0.0659780 & 0.9449513 \\
NA & -0.0129181 & 0.0063493 & 0.0799786 & 0.0953049 & -0.2340004 & 0.1661142 \\
ML & -0.0168983 & 0.0065117 & -0.0736062 & 0.1465186 & 0.0387977 & 0.3267195 \\
\hline & & & $n=1000$ & & \\
\hline QS & -0.0015413 & 0.0005445 & -0.0006907 & 0.0234746 & -0.0136166 & 0.0575812 \\
MCS & -0.0016397 & 0.0005462 & -0.0075067 & 0.0261445 & -0.0003143 & 0.0665571 \\
NA & 0.0022237 & 0.0005151 & 0.1316479 & 0.0084422 & -0.2441716 & 0.0136816 \\
ML & -0.0011093 & 0.0005352 & -0.0116880 & 0.0132224 & 0.0124668 & 0.0286879 \\
\hline
\end{tabular}

with $\alpha, \beta_{0}$, and $\beta_{1}$, while for MCS, $\sigma_{x}^{2}$ is estimated by the empirical variance of the sample values $x_{1}, \ldots, x_{n}$. Both estimates, however, differ only by a negligible amount.

\section{Conclusion}

We proved that in a multivariate mean-variance model, Quasi-Score (QS) is optimal within the class of Linear Score (LS) estimators, in the sense that the ACM of the QS estimator is smaller (in the Loewner order sense) than the ACM of any LS estimator. The QS estimator that we considered is an extended QS estimator, which comprises the estimation of the (nuisance) parameters describing the 
Table 2: The bias and variance of $\alpha, \beta_{0}$ and $\beta_{1}$ when $\sigma_{\delta}^{2}=0.05$

\begin{tabular}{|l|c|c|c|c|c|c|}
\hline & \multicolumn{2}{|c|}{$\alpha$} & \multicolumn{2}{c|}{$\beta_{0}$} & \multicolumn{2}{c|}{$\beta_{1}$} \\
\hline Method & Bias & Var & Bias & Var & Bias & Var \\
\hline
\end{tabular}

$n=100$

\begin{tabular}{|c|c|c|c|c|c|c|}
\hline QS & -0.0196895 & 0.0067698 & -0.0803810 & 0.2183384 & 0.0142384 & 0.5357211 \\
MCS & -0.0273286 & 0.0069701 & -0.1479973 & 0.2983405 & 0.1260944 & 0.7589774 \\
NA & -0.0108817 & 0.0065266 & 0.0551384 & 0.1174278 & -0.1771726 & 0.2180307 \\
ML & -0.0133309 & 0.0067456 & -0.0403318 & 0.1522869 & -0.0083097 & 0.3301717 \\
\hline QS & -0.0022525 & 0.0005487 & -0.0075886 & 0.0169651 & -0.0019069 & 0.0404219 \\
MCS & -0.0036343 & 0.0005601 & -0.0106284 & 0.0192654 & 0.0072325 & 0.0444614 \\
NA & 0.0000362 & 0.0005220 & 0.0869474 & 0.0095582 & -0.1688009 & 0.0186527 \\
ML & -0.0019372 & 0.0005317 & -0.0035439 & 0.0124334 & -0.0056925 & 0.0274534 \\
\hline
\end{tabular}


distribution of the regressor variables.

An important model, where this result can be applied, is the measurement error model given by a mean-variance model in the error-free variables supplemented by a measurement equation, which relates the latent regressor variables to observable variables. In such a model, the parameters describing the distribution of the regressor variables can be considered to be nuisance parameters. In this context, the Corrected Score (CS) estimator, which is a special LS estimator, has been introduced as an alternative to QS. It is well-known that QS is (asymptotically) more efficient than CS, albeit under the assumption that the nuisance parameters are known.

Recently this result has been generalized to the case of unknown nuisance parameters by extending QS in the way indicated above. But this generalization was restricted to a univariate mean-variance model. With the extension to a multivariate model, we are able to analyze the zero-inflated log-linear Poisson measurement error model with a normally distributed regressor variable. (Before this extension, only the ordinary log-linear Poisson measurement error model was amenable to an analysis). In this model, QS is strictly more efficient than CS if the slope parameter is not zero. The mean of the regressor is estimated in the usual way as the arithmetic mean of the observations, but the variance of the regressor must be estimated in a more complicated way taking the complete model into account.

A simulation study confirms these results. 


\section{Acknowledgements}

Support by the German Research Foundation and by the Alexander von Humboldt Foundation is gratefully acknowledged.

\section{References}

[1] Armstrong, B. (1985), Measurement error in the generalized linear model. Communications in Statistics - Simulation and Computation 14, 529-544.

[2] Cameron, A.C. and Trivedi, P.K. (1998), Regression Analysis of Count Data. Cambridge University Press, Cambridge.

[3] Carroll, R. J., Ruppert, D., Stefanski, L.A., and Crainiceanu, C.M. (2006), Measurement Error in Nonlinear Models - A Modern Perspective. $2^{\text {nd }}$ edit., Chapman and Hall, London.

[4] Czado C. and Min A. (2006): Testing for zero-modification in count regression models, Discussion Paper 494, SFB 474, Universität München.

[5] Heyde, C. C. (1997), Quasi-Likelihood And Its Application. Springer, New York.

[6] Kukush, A. and Schneeweiss, H. (2005), Comparing different estimators in a nonlinear measurement error model. I. Mathematical Methods of Statistics 14, 53-79.

[7] Kukush, A., Malenko, A., and Schneeweiss, H. (2006), Optimality of the quasi score estimator in a mean-variance model with applications to measurement error models, Discussion Paper 494, SFB 386, Universität München. 
[8] Lambert, D. (1992), Zero-inflated poisson regression, with an application to defects in manufacturing. Technometrics 34, 1-14.

[9] Nakamura, T. (1990), Corrected score function for errors-in-variables models. Biometrika 77, 127-137.

[10] Schervish, M.J. (1995), Theory of Statistics. Springer, New York.

[11] Stefanski, L. (1989), Unbiased estimation of a nonlinear function of a normal mean with application to measurement error models. Communications in Statistics, Part A - Theory and Methods 18, 4335-4358.

[12] Wedderburn, R.W.M. (1974), Quasi likelihood functions, generalized linear models, and the Gauss-Newton method. Biometrika 61, 439-447. 